\title{
TRATAMIENTO CON DOXAZOSINA EN 3.347 PACIENTES CON SÍNTOMAS DEL TRACTO URINARIO INFERIOR. EFECTO SOBRE SU FUNCIÓN SEXUAL. ESTUDIO IMPROS
}

\author{
C. HERNÁNDEZ FERNÁNDEZ, I. MONCADA IRIBARREN, J. JARA RASCÓN, \\ I. CASTAÑO GONZÁLEZ, M. MORALEJO GÁRATE
}

\author{
Servicio de Urología. Hospital General Universitario Gregorio Marañón. Madrid.
}

Actas Urol Esp. 28 (4): 290-297, 2004

\section{RESUMEN}

TRATAMIENTO CON DOXAZOSINA EN 3.347 PACIENTES CON SÍNTOMAS DEL TRACTO URINARIO INFERIOR. EFECTO SOBRE SU FUNCIÓN SEXUAL. ESTUDIO IMPROS

OBJETIVO: Se realizó un estudio abierto, no comparativo y multicéntrico con el fin de conocer la prevalencia en España de la Disfunción Eréctil (D.E.), asociada a la Hipertrofia Benigna de Próstata (H.B.P.) con sintomatología miccional moderada o severa y constatar la mejoría o desaparición de dicha disfunción como respuesta al tratamiento con $4 \mathrm{mg} /$ día de Doxazosina.

MATERIAL Y MÉTODOS: De un total de 3.901 pacientes reclutados, 331 se excluyeron para el análisis por distintas causas. Del resto, 3.545 tomaron la medicación, y 3.347 completaron todas las visitas del protocolo.

Para su inclusión en el estudio todos debían ser mayores de 40 años, con un I-PSS $>7$, PSA $<4$ y diagnosticados de H.B.P. mediante tacto rectal y ecografia transabdominal.

Se consideró que presentaban algún grado de disfunción eréctil cuando referian tener dificultad en obtener y/o mantener una erección en las cuatro semanas anteriores a la inclusión del paciente en el estudio (preguntas 3 y 4 del Índice Internacional de Función Eréctil).

Todos los pacientes recibieron tratamiento con Doxazosina, con escalada de dosis, $1 \mathrm{mg} /$ día dos semanas, $2 \mathrm{mg} /$ día las dos siguientes y $4 \mathrm{mg} /$ día después hasta completar 6 meses de medicación.

RESULTADOS: La media de pacientes que presentaron disfunción eréctil fue del $69,1 \%$, pasando del $48,3 \%$ en la década entre los 40 y 49 años al 76,3\% en aquellos que se encontraban entre los 60 y 69 años.

En la mayor parte de las regiones españolas la prevalencia de D.E. fluctuó entre el 76,9\% de Cantabria y el 67,2\% de Aragón.

La media de los enfermos en los que desapareció la disfunción eréctil después del tratamiento, con un intervalo de confianza del $95 \%$, fue del $4,5 \%$, variando del $17,5 \%$ en el grupo de edad entre 40 y 49 años y el 1,1\% en los mayores de 70 años.

CONCLUSIONES: La D.E. es un síntoma que se asocia con mucha frecuencia a la H.B.P., incluso en varones relativamente jóvenes.

La Doxazosina parece tener un efecto beneficioso en la mejora de D.E, sobre todo en los pacientes menos añosos.

PALABRAS CLAVE: Hipertrofia prostática. Disfunción eréctil. Doxazosina.

\section{ABSTRACT \\ TREATMENT WITH DOXAZOSIN IN 3347 PATIENTS WITH LOWER URINARY TRACT SYMPTOMS. IMPACT ON SEXUAL FUNCTION. THE IMPROS STUDY}

OBJETIVES: An open, non-comparative, multicenter study was performed to ascertain the prevalence in Spain of erectile dysfunction (ED) associated to benign prostatic hypertrophy (BPH), with moderate to severe urinary symptoms, and to confirm the improvement or disappearance of $\mathrm{ED}$ in response to treatment with $4 \mathrm{mg} /$ day of Doxazosin.

METHODS: Out of the 3901 patients recruited 3545 took the medication and 3347 completed all the protocol visits. BPH patients were considered to have some degree of erectile dysfunction when they reported a difficulty for obtaining and/or maintaining an erection in the four weeks prior to their inclusion in the study (questions 3 and 4 of the International Index of Erectile Function). All the patients were treated with Doxazosin at increasing doses: from $1 \mathrm{mg}$ /day up to $4 \mathrm{mg}$ /day thereafter until 6 months of medication were completed.

RESULTS: The mean number of patients showing erectile dysfunction was $69.1 \%$, ranging from $48.3 \%$ in the $40-49$ years age group to $76.3 \%$ in the group aged from 60 to 69 years. In most Spanish regions, the prevalence of ED ranged from $76.9 \%$ in Cantabria to $67.2 \%$ in Aragon. The mean number of patients in whom erectile dysfunction disappeared after treatment, with a 95\% confidence interval, was $4.5 \%$, ranging from $17.5 \%$ in the $40-49$ age group to $1.1 \%$ in the over 70 s.

CONCLUSIONS: ED is a symptom very often associated to BPH, even in relatively young men. Doxazosin appears to have a beneficial effect in ED improvement, particularly in the younger patients.

KEY WORDS: Prostatic hypertrophy. Doxazosin. Erectile dysfunction. 
$\mathrm{L}$ a obstrucción vesical crónica condiciona una serie de cambios histológicos en la pared vesical que, en algunos pacientes, pueden ser irreversibles, incluso en los periodos más precoces de la enfermedad.

A partir de la $4^{\mathrm{a}}-5^{\mathrm{a}}$ década de la vida, en los varones, la hipertrofia benigna de próstata (HBP) es la responsable de la mayor parte de los sintomas irritativos y/o obstructivos del aparato urinario inferior ${ }^{1}$.

Ya en 1994, la Agencia Americana que se encarga de la política sanitaria y la investigación (Agency for Health Care Policy and Research A.H.C.P.R.), comunicó las líneas maestras en el diagnóstico y tratamiento de la $\mathrm{HBP}^{2}$. Entre sus recomendaciones se incluía la valoración de la sintomatología miccional mediante una batería de preguntas validadas por la Asociación Americana de Urología, mientras que, por su parte, la Organización Mundial de la Salud propició la utilización del I-PSS (International Prostate Symptom Score), que incluye una valoración específica para la calidad de vida del paciente $\mathrm{QL}^{3,4}$.

En la sintomatología miccional se consideran, en relación con su gravedad, tres diferentes niveles según la suma obtenida en la puntuación del I-PSS, <7 sintomatología ausente o leve, entre 819 sintomatología moderada y >20 sintomatología severa.

En cuanto a la QL también se clasifican a los pacientes en tres niveles: $<2$ satisfecho, 3 indiferente y $>3$ insatisfecho.

Ahora bien, al ser los pacientes prostáticos un grupo heterogéneo que engloba una población con diferentes edades, niveles culturales, responsabilidades laborales, etc. Cada vez es más importante considerar, en el momento de indicar un tratamiento, el estado de salud del paciente, la comorbilidad que en ocasiones puede presentar, su tolerancia a ciertos medicamentos, otros fármacos que ya esté tomando $\mathrm{y}$, sin lugar a dudas, las repercusiones que puede tener sobre su actividad sexual.

Si ya es conocido, mediante estudios de tumescencia peneana nocturna, que a partir de los 60 años los varones que aún mantienen relaciones sexuales, van presentando unas erecciones nocturnas cada vez menos frecuentes y dura- deras $^{5}$, es fácil deducir que un alto porcentaje de varones con HBP tendrán, posiblemente asociado al envejecimiento, un alto porcentaje de D.E. que puede verse agravado por los tratamientos empleados para la sintomatología miccional.

Hasta hace poco tiempo este aspecto había sido insuficientemente valorado en la mayor parte de los trabajos sobre alternativas en el tratamiento de la hipertrofia prostática.

Calais Da Silva ${ }^{6}$ en 1997 hacía ya hincapié en la importancia que tiene la sexualidad en la calidad de vida y por lo tanto su importancia a la hora de incluirlo en los distintos cuestionarios a los que nos hemos referido.

La sexualidad es un aspecto complejo en la vida de las personas que en el varón incluye erección, eyaculación, líbido, etc. Por ello es importante especificar a que nos referimos a la hora de evaluar correctamente cualquier estudio.

\section{MATERIAL Y MÉTODOS}

Se analizaron los datos de 3.447 pacientes en un estudio abierto, no comparativo y multicéntrico, llevado a cabo por urólogos de todas las comunidades autónomas de España.

Todos ellos habían acudido espontáneamente a la consulta refiriendo síntomas miccionales que hacían sospechar la existencia de una hipertrofia benigna prostática.

En la primera visita se les practicó una historia clínica, exploración física (incluyendo tacto rectal), análisis de sangre con niveles de PSA, sedimento de orina y ecografía prostática transabdominal.

Así mismo se les entregó el cuestionario I-PSS y el indice internacional de función eréctil IIEF, validado en el VII World Meeting on Impotence celebrado en San Francisco en 1996.

Se incluyeron todos aquellos pacientes diagnosticados de adenoma de próstata que siendo mayores de 40 años tenían un I-PSS $>7$ y un $\mathrm{PSA}<4$ y que firmaron un consentimiento informado para participar en el estudio.

Se consideró que presentaban algún grado de D.E. cuando el varón refería algún problema en obtener y/o mantener una erección durante las 4 últimas semanas previas a su inclusión (preguntas 3 y 4 del cuestionario IIEF).

Se excluyeron, de entrada, aquellos que aún 
siendo diagnosticados de HBP tenían historia previa de síncopes o hipertensión ortostática, accidente cerebrovascular o infarto agudo de miocardio en los últimos tres meses, así como si referian angina de pecho inestable o insuficiencia cardiaca congestiva no tratada.

Tampoco se incluyeron pacientes portadores de sonda vesical, a los que tenían cirugías prostáticas previas ni a los que refirieron hipersensibilidad a los alfa-bloqueantes.

Por último, se descartaron los que por éste $u$ otros motivos ya estaban siendo tratados con alfa o beta bloqueantes, diuréticos, estrógenos, antiandrógenos o antidepresivos tricíclicos en los últimos tres meses y los que recibieron finasteride en los seis meses previos a ser vistos en consulta.

Un total de 3.901 varones fueron enrolados al cumplir los criterios de inclusión y exclusión.

A todos, con o sin D.E., se les trató con una dosis inicial de $1 \mathrm{mg} /$ día de Doxazosina que, de acuerdo al protocolo, se incrementó a las dos semanas a $2 \mathrm{mg} /$ día y dos semanas más tarde a $4 \mathrm{mg} /$ día. Esta última posología se mantuvo hasta completar los 6 meses, siempre y cuando no aparecieran efectos secundarios, en cuyo caso la dosis se redujo en un $50 \%$ o se retiró completamente.

Los pacientes fueron evaluados transcurridos 3 y 6 meses, en ambas revisiones se constató el cumplimiento terapéutico y los acontecimientos adversos, la presión arterial y su frecuencia cardiaca, así como la aparición de enfermedades y tratamientos concomitantes, si los hubo.

En la visita de los 6 meses se les proporcionó de nuevo, para su contestación, los cuestionarios I-PSS e IIEF.

Del número inicial de 3.901 varones incluidos completaron los 6 meses de tratamiento 3.347, lo que supuso el $85,7 \%$, basándose en ellos el estudio de eficacia.

Al mismo tiempo se realizó un estudio de seguridad del fármaco que incluyó también aquellos varones que tomaron al menos una dosis de Doxazosina, este grupo totalizó los 3.570 pacientes, lo que supuso el $91,5 \%$ de los incluidos al inicio.

\section{RESULTADOS}

Al no ser un estudio comparativo no se realizó una valoración estadística convencional. Todos los datos son descriptivos o simplemente comparativos entre ellos mismos, siendo el nivel de confianza del 95\%.

Los varones incluidos se encontraban entre los 40 y 90 años, siendo la media de edad de 64,5 años y la década entre los 60 y 69 años en la que se incluyó un mayor número de pacientes, 1.653 lo que supuso el 46,3\% de la totalidad. La Tabla I muestra dicha distribución.

TABLA I

GRUPOS DE EDAD AL INICIO DEL ESTUDIO

\begin{tabular}{|c|c|}
\hline Edad & $\mathbf{N}^{\circ} \%$ \\
\hline Desconocida & $31(0,9 \%)$ \\
\hline $40-49$ años & $118(3,3 \%)$ \\
\hline $50-59$ años & $810(22,7 \%)$ \\
\hline $60-69$ años & $1653(46,3 \%)$ \\
\hline$>70$ años & $958(26,8 \%)$ \\
\hline
\end{tabular}

En cuanto a las áreas geográficas las que más pacientes incluyeron fueron las de Andalucía y Cataluña con 682 y 555 varones respectivamente, lo que supuso el 19,10\% y el $15,5 \%$ del total respectivamente. En la Tabla II se refleja la distribución geográfica por comunidades autónomas.

En relación con la disfunción eréctil (D.E.) se establecieron dos cohortes, en la primera se incluyeron todos aquellos varones que no presentaban D.E., en el cuestionario inicial, y en la segunda los que sí contestaron tener algún grado de disfunción eréctil.

La distribución por edades demostró un incremento claro de dicha disfunción en relación con la edad de los varones, siendo la media de pacientes que referían problemas en su potencia eréctil del $69,1 \%$, oscilando desde el $48,3 \%$ en la década de los 40 a 49 años, al 76,3\% entre los 60 y 69 años. En el grupo de edad más avanzada (>70) el porcentaje de disfunción fue del 64,5\%, pero hay que tener en cuenta que el $31,9 \%$ o bien no tenía ya ningún intento de relación sexual o simplemente no contestó a ninguna de las dos preguntas del cuestionario.

En la Tabla III se describe la situación basal en cuanto a la D.E. se refiere, y en la Tabla IV dicha distribución por Comunidades Autónomas. 
TABLA II

DISTRIBUCIÓN POR ÁREAS GEOGRÁFICAS

\begin{tabular}{|l|c|c|}
\hline $\begin{array}{l}\text { Comunidad } \\
\text { Autónoma }\end{array}$ & $\begin{array}{c}\text { Número de } \\
\text { pacientes (\%) }\end{array}$ & $\begin{array}{c}\text { Número de } \\
\text { investigadores (\%) }\end{array}$ \\
\hline Andalucía & $682(19,10 \%)$ & $40(19,51 \%)$ \\
\hline Aragón & $134(3,75 \%)$ & $8(3,90 \%)$ \\
\hline Asturias & $131(3,66 \%)$ & $7(3,41 \%)$ \\
\hline Canarias & $170(4,76 \%)$ & $9(4,39 \%)$ \\
\hline Cantabria & $39(1,09 \%)$ & $1(0,48 \%)$ \\
\hline Castilla-La Mancha & $129(3,61 \%)$ & $9(4,39 \%)$ \\
\hline Castilla-León & $159(4 \%)$ & $8(3,9 \%)$ \\
\hline Cataluña & $555(15,5 \%)$ & $34(16,6 \%)$ \\
\hline Comunidad de Madrid & $341(9,6 \%)$ & $19(9,3 \%)$ \\
\hline Comunidad Valenciana & $386(10,8 \%)$ & $25(12,2 \%)$ \\
\hline Extremadura & $98(2,7 \%)$ & $7(3,4 \%)$ \\
\hline Galicia & $272(7,6 \%)$ & $15(7,3 \%)$ \\
\hline Islas Baleares & $61(1,7 \%)$ & $4(2,0 \%)$ \\
\hline La Rioja & $70(2,0 \%)$ & $2(1,0 \%)$ \\
\hline País Vasco & $198(4,6 \%)$ & $10(4,9 \%)$ \\
\hline Comunidad de Murcia & $145(4,1 \%)$ & $7(3,4 \%)$ \\
\hline Total & $3.570(100 \%)$ & $205(100 \%)$ \\
\hline
\end{tabular}

Una vez concluido el periodo de tratamiento con $4 \mathrm{mg} /$ día de Doxazosina durante seis meses, y también con un intervalo de confianza del 95\%, el total de pacientes en los que desapareció la D.E. fue del 4,5\% (3,7\%-5,3\%). Analizando estos resultados por grupos de edad el que alcanzó una mejor respuesta fue el de 40-49, años con un porcentaje de mejoría del $17,5 \%(7,7 \%-27,4 \%)$ y el que consiguió peor respuesta fue el grupo de varones mayores de 70 años, 1,1\% (0,3\%-2,0\%). En la Tabla V puede apreciarse la respuesta en cada uno de los grupos de edad.

Aunque no fue objeto de este trabajo, es importante resaltar que en paralelo se llevó a cabo un estudio de seguridad de Doxazosina en el que se incluyeron todos los varones que al menos habían tomado una dosis del medicamento (3.570) y sólo el 4,5\% de ellos (162 personas) comunicaron algún efecto adverso. De este grupo únicamente el 1,8\% (63) suspendieron la medicación por dichos efectos no deseados. En 88 pacientes, lo que supone el $2,5 \%$, el efecto secundario fue atribuido directamente a la toma del alfa-bloqueante.

\section{DISCUSIÓN}

El urólogo se encuentra en los últimos años ante una decisión compleja cuando tiene que decidir cual es la terapéutica más apropiada en un paciente que refiere sintomatología miccional $\mathrm{y}$ al que le acaba de diagnosticar de un adenoma de próstata.

Las opciones de tratamiento son múltiples y parece lógico utilizar las herramientas que nos ofrece la medicina basada en la evidencia MBE para indicarlas de la mejor manera posible ${ }^{7}$.

No es éste un problema menor ya que el tratamiento de la HBP consume una enormidad de recursos sanitarios. Chicharro y cols. cifran la prevalencia de síntomas urinarios por adenoma de próstata, en la Comunidad Autónoma Andaluza, en el 24,9\% de los varones por encima de los 40 años $^{8}$, cifra que oscila entre el $36,6 \%$ del trabajo de Tsukamoto en Japón ${ }^{9}$ y el 14,2\% del estudio de Sagnier en Francia ${ }^{10}$.

TABLA III

DISTRIBUCIÓN POR EDADES DE LA D.E. AL INICIO DEL ESTUDIO

\begin{tabular}{|l|c|c|c|}
\hline Edad & $\begin{array}{c}\text { Actividad sexual } \\
\text { desconocida o } \\
\text { inexistente (\%) }\end{array}$ & $\begin{array}{c}\text { Si Disfunción } \\
\text { eréctil (\%) }\end{array}$ & $\begin{array}{c}\text { No Disfunción } \\
\text { eréctil (\%) }\end{array}$ \\
\hline Desconocida & $5(16,1 \%)$ & $23(74,2 \%)$ & $3(9,7 \%)$ \\
\hline $40-49$ años & $15(12,8 \%)$ & $57(48,3 \%)$ & $226(27,9 \%)$ \\
\hline $50-59$ años & $76(9,4 \%)$ & $508(62,7 \%)$ & $213(12,9 \%)$ \\
\hline $60-69$ años & $179(10,8 \%)$ & $1.261(76,3 \%)$ & $34(3,6 \%)$ \\
\hline$>70$ años & $306(31,9 \%)$ & $618(64 \%)$ & $522(14,6 \%)$ \\
\hline Total & $581(16,3 \%)$ & $2.467(69,1 \%)$ & \\
\hline
\end{tabular}


DISTRIBUCIÓN POR COMUNIDADES AUTÓNOMAS DE LA D.E. AL INICIO DEL ESTUDIO

\begin{tabular}{|c|c|c|c|}
\hline $\begin{array}{l}\text { Comunidad } \\
\text { autónoma }\end{array}$ & $\begin{array}{c}\text { Actividad sexual } \\
\text { desconocida o } \\
\text { inexistente (\%) }\end{array}$ & $\begin{array}{l}\text { Si Disfunción } \\
\text { eréctil (\%) }\end{array}$ & $\begin{array}{l}\text { No Disfunción } \\
\text { eréctil (\%) }\end{array}$ \\
\hline Andalucía & 57 (4\%) & $519(76,1 \%)$ & $106(15,5 \%)$ \\
\hline Aragón & $38(28,4 \%)$ & $90(67,2 \%)$ & $6(4,5 \%)$ \\
\hline Asturias & $18(13,7 \%)$ & $76(58,0 \%)$ & $37(28,2 \%)$ \\
\hline Canarias & $26(15,3 \%)$ & $123(72,4 \%)$ & $21(12,4 \%)$ \\
\hline Cantabria & $6(15,4 \%)$ & $30(76,9 \%)$ & $3(7,7 \%)$ \\
\hline Castilla-La Mancha & $11(8,5 \%)$ & $90(69,8 \%)$ & $28(21,7 \%)$ \\
\hline Castilla-León & $19(12,0 \%)$ & $124(78,0 \%)$ & $16(10,1 \%)$ \\
\hline Cataluña & $72(13,0 \%)$ & $422(76,0 \%)$ & $61(11,0 \%)$ \\
\hline Comunidad de Madrid & $62(18,2 \%)$ & $238(69,8 \%)$ & $41(12,0 \%)$ \\
\hline Comunidad Valenciana & $54(14,0 \%)$ & $261(67,6 \%)$ & $71(18,4 \%)$ \\
\hline Extremadura & $1(1,0 \%)$ & $92(93,9 \%)$ & $5(5,1 \%)$ \\
\hline Galicia & $43(15,8 \%)$ & $191(70,2 \%)$ & $38(14,0 \%)$ \\
\hline Islas Baleares & $21(34,4 \%)$ & $22(36,1 \%)$ & $18(29,5 \%)$ \\
\hline La Rioja & $50(71,4 \%)$ & $11(15,7 \%)$ & $9(12,9 \%)$ \\
\hline País Vasco & $26(13,2 \%)$ & $142(71,7 \%)$ & $30(15,2 \%)$ \\
\hline Comunidad de Murcia & $77(53,1 \%)$ & $36(24,8 \%)$ & $32(22,1 \%)$ \\
\hline
\end{tabular}

TABLA V

MEJORA DE LA D.E. DESPUÉS DE COMPLETADO EL TRATAMIENTO Y CON UN INTERVALO DE CONFIANZA DEL $95 \%$

\begin{tabular}{|l|c|c|}
\hline $\begin{array}{l}\text { Grupo de } \\
\text { edad }\end{array}$ & $\begin{array}{c}\text { Mejora } \\
\text { en la D.E.\% }\end{array}$ & $\begin{array}{c}\text { Intervalo de } \\
\text { confianza }\end{array}$ \\
\hline $40-49$ años & $17,5 \%$ & $7,7 \%-27,4 \%$ \\
\hline $50-59$ años & $9,1 \%$ & $6,6 \%-11,6 \%$ \\
\hline $60-69$ años & $3,7 \%$ & $2,7 \%-4,8 \%$ \\
\hline$>70$ años & $1,1 \%$ & $0,3 \%-2,0 \%$ \\
\hline Total & $4,5 \%$ & $3,7 \%-5,3 \%$ \\
\hline
\end{tabular}

Sólo analizando el coste en pesetas/año que puede suponer en España el tratamiento de la retención aguda de orina RAO, una de las posibles complicaciones del prostatismo, Gelaber ${ }^{11}$ la cifra entre los mil y dos mil quinientos millones de pesetas (entre 6 y 15 millones de $€$ ).

Dentro del terreno de la farmacopea, el tratamiento médico de la HBP se apoya en tres familias de fármacos: alfa-bloqueantes, inhibidores de la 5-alfa-reductasa y fitoterapia.
Del primer grupo tenemos evidencias, tanto en estudios comparados con placebo como en trabajos donde se comparan unos con otros, que la eficacia de las distintas moléculas es similar en la mejora de la sintomatología miccional secundaria al adenoma de próstata ${ }^{12-15}$. Está demostrado que los alfa-bloqueantes reducen los sintomas urinarios y mejoran la calidad de vida de los pacientes prostáticos.

La única diferencia constatable se encuentra en que los alfa-bloqueantes específicos de los receptores $1 \mathrm{~A}$ y $1 \mathrm{D}$, que se encuentran fundamentalmente en el aparato urinario inferior, condicionarian menores efectos hipotensores, sobre todo en los pacientes de mayor edad ${ }^{16}$.

En nuestro estudio se recogieron un 1,1\% de efectos secundarios en el sistema cardiovascular y la caída media de la tensión arterial fue de 6,6 $\mathrm{mm} \mathrm{Hg}$ en la presión sistólica y de 4,3 $\mathrm{mm} \mathrm{Hg}$ en la diastólica, alteraciones tensionales que en la mayoría de los pacientes no condicionaron ningún efecto adverso. 
La investigación básica nos sigue aportando datos en cuanto a los alfa-receptores y sus distintos subtipos $\alpha 1 \mathrm{~A}, \alpha 1 \mathrm{~B}, \alpha 1 \mathrm{D}, \alpha 1 \mathrm{~L}$, etc. ${ }^{17}$, ello nos hace esperar la aparición de moléculas cada vez más selectivas, pero también perder la oportunidad de que un solo fármaco pueda ser también beneficioso para otras patologías, que con frecuencia coinciden con una alta prevalencia, en el varón añoso (hipercolesterolemia, hipertensión, etc.).

Uno de los problemas ante los que se encuentra la población, a partir de una cierta edad, es la necesidad de tomar un número excesivo de fármacos para controlar la multipatología que en muchas ocasiones presentan (hipertensión, hipercolesterolemia, osteoporosis, hipertrofia benigna de próstata, etc.) y por lo tanto la posibilidad de utilizar principios activos que sean capaces de actuar a distintos niveles puede convertirse en una gran ventaja.

La hipertensión es una enfermedad habitual en la mayor parte de las sociedades occidentales, variando su prevalencia según la definición empleada. En la actualidad tomando como límite normal las cifras 140/90 mm Hg, existen datos para conocer, que a partir de los 60 años, más del $60 \%$ de las personas son hipertensas. La prevalencia aumenta claramente con la edad.

Esta enfermedad junto a la hipercolesterolemia y al consumo de tabaco son los tres principales factores de riesgo de la cardiopatía isquémica y el principal en cuanto a los accidentes cerebrovasculares.

Desde un enfoque de incidencia y prevalencia lo mismo ocurre con la HBP, pasando de una tasa del 138 por mil entre los varones de 40 a 49 años al 430 por mil en la década de los 60 a los 69 años.

Dentro de la valoración de la calidad de vida, ésta es entendida de forma distinta por cada paciente, y dentro de ella, la sexualidad, es muy poco valorada en la mayoría de los trabajos publicados sobre tratamiento médico de la HBP, siendo éste un aspecto fundamental en la vida de muchos de estos varones y que por lo tanto ha de ser tenido en cuenta a la hora de indicar una $u$ otra alternativa terapéutica ${ }^{18}$.

Es además imprescindible definir, dentro del tema de la sexualidad, que es lo que valoramos en cualquier estudio, trastornos de la erección, eyaculación, disminución de la líbido, grado de satisfacción sexual, etc.

Con el fin de sistematizar la valoración de estos síntomas se validó en 10 idiomas el International Index of Eréctil Function IIEF y que incluye preguntas para todas estas áreas de la esfera sexual.

En las preguntas número 3 "durante las cuatro últimas semanas cuando intentó usted realizar el acto sexual”, ¿con qué frecuencia fue usted capaz de penetrar a su pareja? y la número 4 "durante las cuatro últimas semanas durante el acto sexual”, ¿con qué frecuencia fue usted capaz de mantener la erección después de haber penetrado a su pareja?, se identifican alteraciones en relación con la erección.

En nuestro estudio sólo el 14,6\% de los varones contestaron a las dos preguntas con la respuesta "siempre o casi siempre", en el 69,1\% la respuesta elegida fue alguna de las otras cuatro opciones, mientras que el $4,5 \%$ no referían tener ningún tipo de actividad sexual.

Es importante destacar que en el grupo de edad más joven (40-49 años), de un total de 118 pacientes, ya el 48,3\% de ellos comunicaban un mayor o menor grado de disfunción eréctil.

En la literatura médica existen datos que nos permiten conocer como afectan los inhibidores de la 5-alfa reductasa a la erección de los pacientes $^{19,20}$, mientras que cuando se revisan los trabajos sobre alfa-bloqueantes los datos que, se han recabado sobre la sexualidad de los varones, están fundamentalmente relacionados con la eyaculación y no tanto con la erección ${ }^{12,13,21,22}$.

Sin embargo se citan de forma repetida los resultados del estudio Tomhs ${ }^{23}$, donde pacientes hipertensos fueron tratados con Doxazosina y los datos de mejora de la D.E. no eran uno de los objetivos del estudio ni se validaron correctamente, además de que el número total de pacientes incluidos fue de 21 , ocho tratados con Doxazosina y trece en el grupo placebo.

Es por ello que han de realizarse más estudios en este sentido como apuntan Zlotta y Schulman ${ }^{24}$.

Sí conocemos por distintos trabajos que los alfa-receptores son fundamentales en la respuesta eréctil ${ }^{25,26}$, incluso en estudios experimentales 
se demuestra que la destrucción del locus ceruleus, núcleo que contiene abundantes neuronas con receptores noradrenérgicos, condiciona una disminución del $30 \%$ de la noradrenalina del cerebro y la aparición de priapismo en algunos animales ${ }^{27}$. De forma contraria, si se inyecta clonidina (alfa-2 agonista) en el cerebro de las ratas se inhibe su erección.

No es descabellado aseverar que en la HBP, en la hipertensión arterial y en la disfunción eréctil, existe una hiperactividad del sistema simpático, de ahí que la utilización de un alfa-bloqueante suponga un efecto beneficioso en estas tres patologías, todas ellas con una alta prevalencia en el varón a partir de la $4^{\mathrm{a}}-5^{\mathrm{a}}$ década de la vida.

También es lógico asumir que, cuanto mayor es la edad del paciente, esta hiperactividad adrenérgica jugará un menor papel en estas tres patologías y serán las alteraciones estructurales en próstata, arterias y cuerpos cavernosos las que jueguen un papel más importante en la evolución de dichas enfermedades. Probablemente por ello la eficacia de los alfa-bloqueantes en la mejora de la $\mathrm{DE}$, como se demuestra en nuestro estudio, es mayor cuando se utilizan en los varones más jóvenes, $17,5 \%$ en la década de los 40-49 años frente al 1,1\% de mejora en los mayores de 70 años.

La Doxazosina es un antagonista alfal, con una prolongada vida media, lo cual permite una sola toma al día. Su tolerancia es buena, sólo el $1,8 \%$ de nuestros pacientes suspendieron la medicación por efectos adversos, pero con el fin de disminuir el posible ortostatismo se recomienda iniciar la terapéutica de forma escalonada, aumentando paulatinamente la dosis.

La aparición de la nueva formulación GITS (Gatrointestinal Therapeutic System) evita esta limitación puesto que modifica el tiempo que el fármaco tardó en alcanzar su concentración máxima, así como su valor absoluto, pero sin modificar la biodisponibilidad del mismo ${ }^{28}$.

Es por tanto lógico suponer que, también en la $\mathrm{DE}$, la nueva formulación tendrá un efecto similar a la Doxazosina standard, aunque se precisa de estudios concretos que lo corroboren.

\section{CONCLUSIONES}

El impacto en la esfera sexual de cualquier tratamiento en la HBP ha de ser tenido en cuenta, puesto que afecta de forma importante a la calidad de vida de un elevado número de varones.

La Doxazosina (alfal antagonista) a dosis de 4 $\mathrm{mg} /$ día puede, sobre todo en los pacientes prostáticos más jóvenes, mejorar la D.E. si es que la presentan.

La Doxazosina a dosis de $4 \mathrm{mg} /$ día es un fármaco bien tolerado y con escasos efectos secundarios.

La nueva formulación (Doxazosina GITS) es de suponer que consiga el mismo efecto, con la ventaja de poder evitar la posología mediante escalada de dosis.

\section{REFERENCIAS}

1. CHUTE CG, PAUSER LA, GIRMAN CJ, OESTERLING JE, GUESS HA, JACOBSEN SJ, LIEBER MM.: The prevalence of prostatism: a population based survey of urinary symptoms. J Urol 1993; 150: 85-89.

2. ROEHRBORN CG.: The newly established guidelines for the diagnosis and management of benign prostatic hyperplasia. Curr Opin Urol 1995; 5: 30-34.

3. DENIS L, MCCONNELL J, KHOURY S, ABRAMS P, BARRY M, BARTSCH G, COCKETT A, CORRIERE J, DREIKORN K, GIBBONS R, GRIFFITHS K, HOLD T, HOLTGREWE L, JARDIN A, KOYANAGI T, MEBUST W, MURPHY G, ROEHRBORN C, SMITH P, and Members of the Committees: $4^{\text {th }}$ International Consultation on BPH. Recommendations of the International Scientific Committee: the evaluation and treatment of lower urinary tract symptoms (LUTS) suggestive of benign prostatic obstruction; in Denis L, Griffith K, Khoury S, Cockett ATK, McConnell J, Chatelain C, Murphy G, Yoshida O. (eds): proceedings of the 4th International Consultation on Benign Prostatic Hyperplasia (BPH). Plymouth, Plymbridge Distributors, 1998.

4. VELA NAVARRETE R, MARTÍN MORENO JM, CALAHORRA FJ, DAMIÁN MORENO J, HERNÁNDEZCORONADO A, BOYLE P.: Validación cultural y lingüística en castellano del baremo internacional de sintomas prismáticos (I-PSS). Actas Urol Esp 1994; 18: $841-847$.

5. SCHIARI SE.: Nocturnal penile tumescence in healthy aging men. J Gerontology 1988, 43: 146-150.

6. CALAIS DA SILVA F, MARQUIS P, DESCHASEAUX P, GINESTE JL, CAUQUIL J, PATRICK DL.: Relative importance of sexuality and quality of life in patients with prostatic symtoms. Results of an international study. Eur Urol 1997; 3 (3): 272-280.

7. POSTIUS ROBERT J, CASTRO DÍAZ D.: Tratamiento farmacológico de la hiperplasia benigna de próstata basado en la evidencia. Rev Clin Esp 1999; 199 (2): 58-61.

8. CHICHARRO MOLERO JA, BURGOS RODRÍGUEZ R, SÁNCHEZ-CRUZ JJ, DEL ROSAL SAMANIEGO JM, RODERO GARCIA P, RODRÍGUEZ VALLEJO JM.: Prevalence of BPH in Spanish men aged above 40 years. J Urol 1998; 159: 878-882. 
9. TSUKAMOTO T, KUMAMOTO Y, MASUMORI N, MIYAKE H, RHODES T, GIRMAN CJ, GUESS HA, JACOBSEN SJ, LIEBER MM.: Prevalence of prostatism in japanese men in a community based study with comparison to a similar American Study. $J$ Urol 1995; 154: 391-396.

10. SAGNIER PP, MCFARLANE G, RICHARCH F, BOTTO H, TEILLAC C, BOYLE P.: Results of an epidemiological survey using a modified American Urological Association symptom index for benigm prostatic hyperplasia in France. J Urol 1994; 151: 1266-1272.

11. GELABERT MAS A.: Retención aguda de orina: un problema de salud pública. Rev Clin Esp 1999; 199 (2): 24-29.

12. JADIN A, BESADOUM H, DELAUCHE-LAVALLIER MC, ATTALI P, and the BPH ALF Group: long-term treatment of benign prostatic hyperplasia with alfuzosin: a 24-30 month survey. $\mathrm{Br} J$ Urol 1994; 74: 759-584.

13. LEPOR H, for the Terazosin Research Group: Long term efficacy an safety of terazosin in patients with benign prostatic hyperplasia. Urology 1995; 45: 406-413.

14. LEPOR H, KAPLAN SA, KLIMBERG I, MOBLEY DF, FAWZY A, GAFFNEY M, ICE K, DIAS N, for the Multicenter Study Group: Doxazosine for benign prostatic hyperplasia: long term efficacy and safety in hypertensive and normotensive patients. J Urol 1997; 157: 525-530.

15. SCHULMAN CC, LOCK TMTW, BUZELIN JM.: Tamsulosi: 3-year follow-up of efficacy and safety in 516 patients with LUTS sugestive of BPH. J Urol 1998; 159 (suppl): 256 (abst. 983).

16. DEMEY C, MICHEL MC, MCEVEN J, MORELAND T.: A double-blind comparison of terazosin and tamsulosin on their differential effects on ambulatory blood pressure and nocturnal orthostatic stress testing. Eur Urol 1998; 33 (5): 481-488.

17. HIEBLE JP, RUFFOLO RR Jr.: Recent advances in the identification of $\alpha 1$ and $\alpha 2$ adrenoceptor subtypes: therapeutic implications expert opinion on Investigational Drugs 1997; 6: 367-387.

18. SCHOU J, HOLM NR, MEYHOFF HH.: Sexual function in patients with symtomatic benign prostatic hyperplasia. Scand J Urol Nephrol Suppl 1996; 179: 112-119.

19. MARBERGER MJ.: Long-term effects of finasteride in patients with benign prostatic hyperplasia: a double-blind, placebo-controlled, multicenter study. Urology 1998; 51: 677-686.

20. MCCONNELL JD, BRUSKEWITZ R, WLSH P, ANDRIOLE G, LIEBER M, HOLTGREWE HL, ALBERTSEN P, ROEHRBORN CG, NICKEL JC, WANG DL, TAYLOR AM, WALDSTREICHER J.: The effect of finasteride on the risk of acute urinary retention and the need for surgical treatment among men with benign prostatic hyperplasia. New Engl J Med 1998; 338: 557-563.
21. LUKACS B, LEPĖGE A, THIBAULT P, JADIN A.: Prospective study of men with clinical benign prostatic hyperplasia treated with alfuzosin by general practitioners: 1-year results. Urology 1996; 48: 731-740.

22. FAWZY A, BRANN K, LEWIS GP, GAFFNEY M, ICE K, DIAS N, for the Multicenter Study Group: Doxazosin in the treatment of benign prostatic hyperplasia in normotensive patients: a multicenter study. J Urol 1995; 154: 105-109.

23. GRIM RH, GRANDITS GA, PRINEAS RJ, MCDONALD RH, LEWIS CE, FLACK JM, YUNIS C, SVENDSEN K, LIEBSON PR, ELMER PJ, STAMLER J, for the Tomhs Research Group: long-term effects on sexual function of five antihypertensive drugs and nutritional hygienic treatment in hypertensive men and women. Treatment of mild hypertension study (Tomhs). Hypertension 1997; 29: 8-14.

24. ZLOTTA AR, SCHULMAN CC.: BPH and sexuality. Eur Urol 1999; 36 (suppl. 1): 107-112.

25. BECKER AJ, STIEF CG, MACHTENS S, SCHULTHEISS D, HARTMANN U, TRUSS MC, JONAS U.: Oral phentolamine as treatment for erectile dysfunction. $J$ Urol 1998; 159: 1214-1216.

26. ERUS E, PITTLER MH.: Yohimbine for erectil dysfunction: a systematic review and metanalysis of randomized clinic trials. J Urol 1998; 159: 433436.

27. ROUSSEL B, PUJAL JF, JOUVET M.: Effects of lesions in the pontine tegmentum on the sleep stages in the rat. Arch Ital Bid 1976; 114: 188209.

28. CHUNG M, VASHI V, PUENTE J, SWEENEY M, MEREDITH P.: Clinical pharmacokinetics of Doxazosin in a controlled-release gastrointestinal therapeutic system (GITS) formulation. $\mathrm{Br} \mathrm{J}$ Clin Pharmacol 1999, 48: 678-687.

29. GRATZKE P, KIRBY S.: Doxazosin GITS and doxazosin standard in patients with Benign Prostatic Hyperplasia. Fortschritte der Medizin Journal 2000; 11: 83-92.

30. KIRBY RS, ANDERSEN M, GRATZKE P, DAHLSTRAND C, HOYE K.: A combined analysis of double-blind trials of efficacy and tolerability of doxazosin-gastrointestinal therapeutic system, doxazosin standard and placebo in patients with benign prostatic hyperplasia. BJU International 2001; 87: 192-200.

Dr. C. Hernández Fernández

C/ Rafael Bergamín, 9 - 12 F

28043 Madrid

(Trabajo recibido el 27 septiembre de 2002) 